\title{
Passive neutron coincidence counting with plastic scintillators for the characterization of radioactive waste drums
}

\author{
B. Simony, B. Pérot, C. Carasco, F. Jallu, N. Saurel, S. Colas, P. Girones, J. Collot
}

\begin{abstract}
This paper reports a feasibility study, performed by numerical simulation with MCNPX PoliMi v2.0, for plutonium quantitative assessment in radioactive waste packages by passive neutron coincidence counting with plastic scintillators. Owing to their low cost and good detection efficiency for fast neutrons, plastic scintillators could indeed constitute a good alternative to ${ }^{3} \mathrm{He}$ proportional counters, which have become too costly because of ${ }^{3} \mathrm{He}$ global shortage. However, their highsensitivity to gamma rays and cross talk are well-known drawbacks that need to be carefully studied. A measurement system for $118 \mathrm{~L}$ drums filled either with metallic or organic technological wastes has been modeled with MCNPX PoliMi, and output data have been processed with ROOT. A $5 \mathrm{~cm}$ thick lead shield is used in front of the detectors to attenuate plutonium and americium gamma radiation. In the studied cases, triple coincidences due to ${ }^{240} \mathrm{Pu}$ spontaneous fissions represent more than $85 \%$ of the total signal when using cross-talk rejection algorithms, the $15 \%$ remaining coincidences being due to parasitic coincidences caused by $(\alpha, n)$ reactions or Am and Pu gamma rays. Although cross-talk rejection significantly reduces counting statistics, a few thousand triple coincidences are still recorded for $\mathbf{1 ~ g ~ o f ~} \mathrm{Pu}$ homogeneously distributed in both metallic and organic matrices in a $25 \mathrm{~min}$ acquisition. For higher masses of $\mathrm{Pu}$, a linear evolution of the number of coincidences with the mass is observed up to about $10 \mathrm{~g}$. In addition, a study of $\mathrm{Pu}$ localization effects has shown that the triple coincidence difference is smaller than $20 \%$ between a point-like fission source and the homogeneous distribution in the drum.
\end{abstract}

Index Terms - Passive neutron coincidence counting, plastic scintillators, nuclear waste drums, cross talk, MCNPX-PoliMi, ROOT

Manuscript submitted on April $12^{\text {th }}, 2017$. This work was supported by the CEA R\&D program for radioactive waste characterization.

Benoît Simony , Bertrand Pérot, Cédric Carasco and Fanny Jallu, CEA, DEN, Cadarache, Nuclear Measurement Laboratory, F-13108 Saint-Paul-lezDurance, France.

Nicolas Saurel and Sébastien Colas, CEA Valduc, F-21120 Is-sur-Tille, France.

Philippe Gironès, CEA, DEN, Marcoule, 30207 Bagnols-sur-Cèze, France.

Johann Collot, Laboratory of Subatomic Physic and Cosmology, Université Grenoble Alpes, CNRS/IN2P3, France.

\section{INTRODUCTION}

In the fields of safeguards, of criticality control of nuclear processes, of dismantling of nuclear facilities, and of radioactive waste characterization, the quantification of nuclear materials is essential. In usual passive neutron coincidence counting (PNCC), this goal is achieved by recording coincidence pairs induced by spontaneous fission using ${ }^{3} \mathrm{He}$ gas counters, which are very sensitive to thermalized neutrons but not to gamma rays up to a dose rate around $0.1 \mathrm{~Gy} \cdot \mathrm{h}^{-1}$ [1]. However, due to ${ }^{3} \mathrm{He}$ gas global shortage, the cost of these detectors has become prohibitive, which has motivated research programs to find alternative detectors [2]. Liquid organic scintillators are interesting, since they allow neutron - gamma pulse shape discrimination (PSD) [3]. However, since PSD exploits scintillation photons that form the pulse tail, at high count rate, pulse pile-up can decrease the PSD figure of merit. Also, PSD scintillators are expensive and using a large amount of scintillation liquid in a $118 \mathrm{~L}$ waste drum PNCC would be almost as expensive as ${ }^{3} \mathrm{He}$ detectors. In contrast, standard plastic scintillators allow very fast counting, and covering a large detection surface at reasonable cost. Note that plastic scintillators with PSD capabilities have been recently developed and used in safeguards applications [4,5], but as for liquid scintillators, their use for PNCC of radioactive waste packages would require a large number of small detectors, making again the system costly, and would also be limited in terms of count rate (to maintain acceptable PSD features).

Our previous work has shown that plutonium could be measured by PNCC in $118 \mathrm{~L}$ technological, metallic waste drums using basic EJ-200 plastic scintillators, without PSD capability, but shielded by $10 \mathrm{~cm}$ of lead $[6,7]$ to protect against most of the gamma rays coming from the waste. A characterization of cross-talk occurring in these plastic scintillators has also been presented, showing that most of cross-talk-induced coincidences imply two pulses separated by less than $20 \mathrm{~ns}$ and $20 \mathrm{~cm}$ between neighbor detectors. Using a $10 \mathrm{~cm}$ lead shield allows neglecting gamma-rays emissions with a comfortable margin. However, the measurement cell mass exceed the maximum mass allowed in some of the facilities in which passive neutron coincidence counting is foreseen.

Taking into account this practical constraint, we thus report in this paper further numerical simulations using a thinner, $5 \mathrm{~cm}$ lead shield, in an attempt to limit the weight and the cost of the system. Using a thinner lead shield also increase the number of coincidences and reduce counting statistics 
uncertainties. Parametric studies are performed, by varying the nature of the waste matrix (organic or metallic), the mass of $\mathrm{Pu}$, or studying the impact of $\mathrm{Pu}$ localization inside the drum, which was not done in [7], considering no other neutron/gamma emitters than plutonium and americium (i.e. no trace of fission or activation products). Cross-talk rejection is also studied, consisting in freezing the close neighbors of a primary triggered detector during $10 \mathrm{~ns}$. In this way, coincidences due to neutron or gamma scattering in adjacent scintillators can be discarded. The difference of composition of triple coincidences in terms of neutron and gamma-ray pulses, before and after cross-talk rejection, is also analyzed to better understand the effect of cross-talk rejection.

\section{NUMERICAL SIMULATION}

A standard $118 \mathrm{~L}$ technological waste drum is homogeneously filled with a $0.5{\mathrm{~g} . \mathrm{cm}^{-3}}^{-3}$ stainless steel matrix, or with a $0.2 \mathrm{~g} . \mathrm{cm}^{-3}$ cellulose matrix. The low density of the metallic matrix represents an average value for a drum containing metallic components occupying a small volume. A $5 \mathrm{~cm}$ thick lead screen surrounds the drum to limit the gamma-ray flux coming from radioactive decays of $\mathrm{Pu}$ and ${ }^{241} \mathrm{Am}$ and reaching the detectors, compared to the $10 \mathrm{~cm}$ thick lead screen considered in previous work [6,7]. The detection system is composed of 19 plastic scintillators, as shown in Fig. 1. The vertical detectors are $20 \mathrm{~cm}$ wide, $10 \mathrm{~cm}$ thick, and $100 \mathrm{~cm}$ high. The top and bottom horizontal detectors show the same width and thickness, but they are $83 \mathrm{~cm}$ or $57 \mathrm{~cm}$ long, depending on their position.

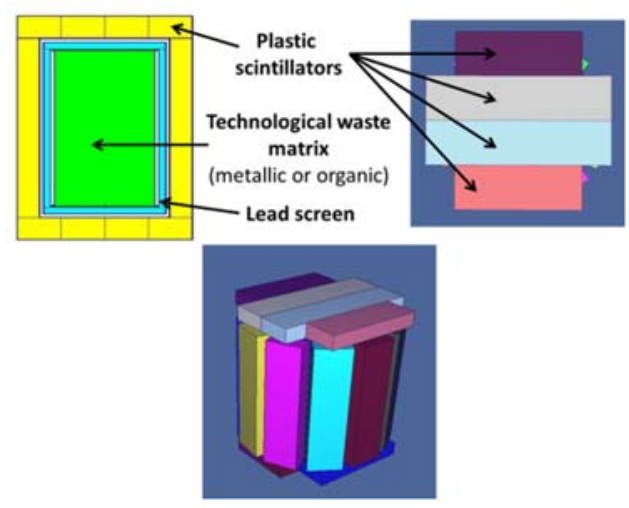

Fig. 1. MCNPX model of a $118 \mathrm{~L}$ waste drum surrounded by a lead shield and plastic scintillators.

A typical plutonium isotopic composition with a large fraction of ${ }^{241} \mathrm{Am}$ (radioactive waste with a long storage period) has been modeled, see Tab I, in order to take into account a large background in terms of $(\alpha, n)$ reactions. Three sources of events are modeled with MCNPX-PoliMi [8]. This allows us to simulate correlated neutron-gamma emissions [9], as would appear in radioactive materials, see Table II. The first source is a ${ }^{240} \mathrm{Pu}$ spontaneous fission (SF) source that emits prompt fission neutrons correlated to prompt gamma rays. The second source is an $\mathrm{AmO}_{2}(\alpha, n)$ source that emits a neutron correlated with gamma rays consecutive to the de-excitation of the daughter nucleus, such as the $1634 \mathrm{keV}$ line of ${ }^{20} \mathrm{Ne}$ or the
$2438 \mathrm{keV}$ ray of ${ }^{21} \mathrm{Ne}$. The third source is a gamma-ray source that takes into account gamma emissions after alpha or beta disintegration of $\mathrm{Pu}$ and $\mathrm{Am}$ isotopes, which was not the case in our previous study with a $10 \mathrm{~cm}$ thick lead screen [7], as they were totally absorbed. In order to limit the computation time, photons susceptible to be strongly attenuated by the $5 \mathrm{~cm}$ lead shield, i.e. with energy below $200 \mathrm{keV}$, have not been modeled. Note that this $3^{\text {rd }}$ gamma source emits single (uncorrelated) photons, which can however produce coincidences due to Compton scattering between different detectors (cross talk).

TABLE I. ISOTOPIC COMPOSITION OF THE MIXTURE OF PU AND 241AM CONSIDERED FOR SIMULATION. POURCENTAGE OF ${ }^{241}$ AM CORRESPONDS TO THE RATIO $\mathrm{M}\left({ }^{241} \mathrm{AM}\right) / \mathrm{M}(\mathrm{PU})$.

\begin{tabular}{|c|c|}
\hline ISOTOPE & $\begin{array}{c}\text { ISOTOPIC } \\
\text { COMPOSITION }\end{array}$ \\
\hline${ }^{238} \mathbf{P U}$ & $2 \%$ \\
${ }^{239} \mathbf{P U}$ & $59 \%$ \\
${ }^{240} \mathbf{P U}$ & $27 \%$ \\
${ }^{241} \mathbf{P U}$ & $3 \%$ \\
${ }^{242} \mathbf{P U}$ & $9 \%$ \\
${ }^{241} \mathbf{A M}$ & $8 \%$ \\
\hline
\end{tabular}

TABLE II. MCNPX POLIMI SOURCE CARDS AND REACTION RATES USED IN SIMULATIONS. REACTION RATES ARE REFERRED PER GRAM OF THE MIXTURE OF PU AND ${ }^{241}$ AM CONSIDERED

\begin{tabular}{|c|c|c|}
\hline SOURCE & $\begin{array}{c}\text { MCNPX PoliMi source } \\
\text { card used }\end{array}$ & $\begin{array}{c}\text { Reaction rate } \\
\left(\mathbf{s}^{-1} \cdot \mathbf{g}_{\mathbf{P u}^{-1}}\right)\end{array}$ \\
\hline SPONTANEOUS FISSION (SF) & IPOL $(1)=3\left({ }^{240} \mathrm{PU}\right)$ & 224 \\
(ALPHA,N) REACTIONS & $\operatorname{IPOL}(1)=-41\left(\mathrm{AMO}_{2}\right)$ & 570 \\
$\gamma$ RAYS OF PU AND AM & $\begin{array}{c}\text { IPOL }(1)=0(\gamma \text { RAY }>200 \mathrm{KEV} \\
\left.\text { OF ISOTOPES OF PU AND }{ }^{241} \mathrm{AM}\right)\end{array}$ & 404,174 \\
\hline
\end{tabular}

One calculation is performed for each source with MCNPX PoliMi to create three sets of histories associated to (1) ${ }^{240} \mathrm{Pu}$ spontaneous fission correlated particles, (2) $\mathrm{AmO}_{2}(\alpha, n)$ correlated neutron and gamma particles, and (3) pulses due to the uncorrelated gamma source. Post-processing algorithms have been developed with ROOT data analysis software [10] to produce realistic pulse trains. For all pulses related to the same source event, which by definition belong to the same history, a macroscopic time offset is sampled between 0 and $\mathrm{T}, \mathrm{T}$ being the total acquisition time (minutes or hours), and added to the MCNP-PoliMi time (nanoseconds) of the events. In this way, all histories are made to happen randomly during the whole measurement time, which allows creating random coincidences. Fig. 2 illustrates the way pulses from different sources are mixed, allowing random coincidences to happen. Within the 100 ns time gate triggered by a pulse recorded in any detector, other pulses are searched in the other detectors allowing in this way to identify coincidences of multiplicity one (i.e. doubles), two (triples), or three (quadruples) when one, two or three other scintillators have been triggered. Such coincidence counting is performed for the randomized pulse train, but also for each history related to the different sources, which allows us to estimate the proportion of accidental coincidences. In order to limit the contribution of cross talk, calculations are also performed by rejecting the pulses of the closest neighbors over $10 \mathrm{~ns}$, because cross-talk events involving photons and neutrons mostly occur in this time range [7]. 


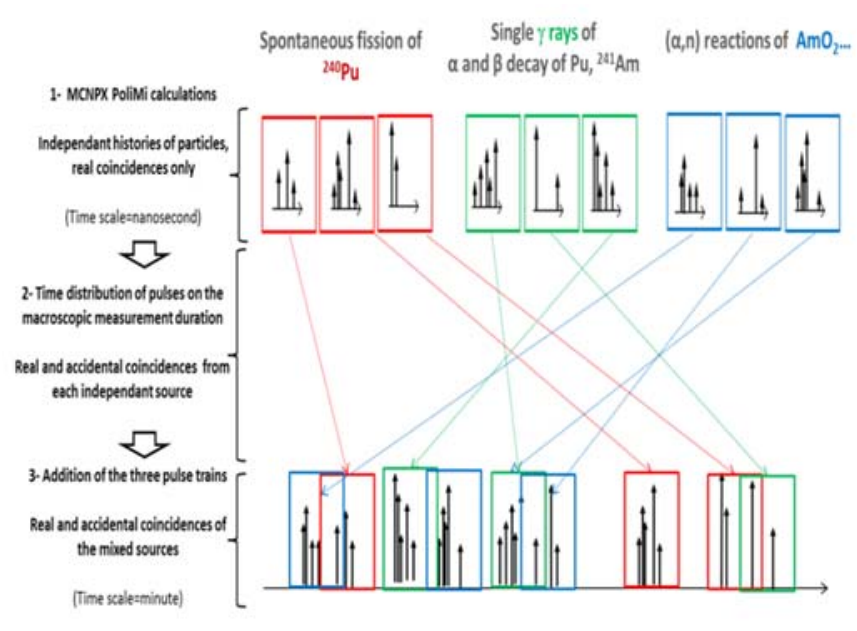

Fig. 2. Post processing of MCNPX PoliMi histories to create realistic pulse trains.

\section{RESULTS AND DISCUSSION}

Tables III and IV present the number of coincidences recorded for the metallic matrix, without and with cross-talk rejection, respectively, and Tables V and VI for the organic matrix. In addition, Fig. 3 and 4 present the composition of real triples of ${ }^{240} \mathrm{Pu}$ and $(\alpha, \mathrm{n})$ reactions of $\mathrm{AmO}_{2}$ in the metallic and organic matrices, respectively.

Although cross-talk rejection penalizes the counting statistics, it significantly improves the fraction of doubles and triples due to $\mathrm{Pu}$ spontaneous fissions, with respect to those due to $(\alpha, \mathrm{n})$ reactions, as can be seen when comparing Tables II and III for the metallic matrix, Tables IV and V for the organic matrix, and in further Table VI as a function of Pu mass. And even after cross talk rejection, the number of triples remains quite large, i.e. more than 7000 in Table III for the metallic matrix, and close to 2000 in Table $\mathrm{V}$ for the organic matrix. By comparison with a $10 \mathrm{~cm}$ thick lead screen, the number of triple coincidences after cross-talk rejection was 2400 for the metallic matrix and less than 400 with the organic matrix [7]. These differences on statistics of a factor of 3.5 and 5 for metallic and organic matrix, respectively, are due to the lower gamma-ray attenuation and the better detection efficiency of neutron by the $5 \mathrm{~cm}$ vs. $10 \mathrm{~cm}$ lead shield. Indeed, prompt fission gamma-ray pulses constitute a significant part of $\mathrm{Pu}$ triple coincidences as shown further in Fig. 3 and 4. Also, neutron detection efficiency of the system with an empty drum has been estimated to be $23.9 \%$ with a $5 \mathrm{~cm}$ thick lead shield and decrease to $18.3 \%$ with $10 \mathrm{~cm}$ of lead, since neutrons can scatter in the lead and lose enough energy to fall below the $100 \mathrm{keV}$ detection threshold. Given that detection efficiency of triple coincidences only composed by neutrons ('nnn' coincidences, as explained further) is proportional to the cube of the neutron detection efficiency, the $30 \%$ increase of the neutron detection efficiency improves the statistics of triple neutron coincidences by about a factor 2 .

The selectivity of the method to $\mathrm{Pu}$, with respect to $\mathrm{AmO}_{2}$ or other possible sources of correlated $(\alpha, n)$ neutrons and gamma rays, can be assessed by the fraction of triples due to spontaneous fissions of ${ }^{240} \mathrm{Pu}$ in the total amount of triples. For the metallic matrix, it can be calculated from Table III that this ratio is larger than $95 \%$ and, after cross talk rejection, on can see from the data in Table IV that this ratio increases to nearly $100 \%$, triples due to $(\alpha, n)$ reactions being almost entirely rejected. The same effect is observed for the organic matrix: in Table $\mathrm{V}$, the ratio is larger than $87 \%$, and it approaches $100 \%$ in Table VI, after cross-talk rejection, which was also observed with a $10 \mathrm{~cm}$ think lead screen [7]. Equivalent performances in terms of Pu selectivity can hence be obtained even by reducing the amount of lead surrounding the drum, because cross-talk rejection allows eliminating a strong amount of coincidences due to gamma-ray cross talk. Uncertainties presented in Table III to VI are counting statistics of coincidences recorded with MCNPX PoliMi calculations. Experiments are underway to validate the performances, which will also allow estimating other causes of uncertainties related to simulation biases due to modeling geometrical simplifications or nuclear data by performing a calculation vs. experiment comparison.

TABLE III. CALCULATED COINCIDENCES FOR THE METALLIC MATRIX WITH A 5 CM THICK LEAD SCREEN, FOR A 25 MINUTES MEASUREMENT TIME AND $1 \mathrm{G}$ OF PU, WITHOUT CROSS-TALK REJECTION.

\begin{tabular}{|c|c|c|c|c|}
\hline Source type $\rightarrow$ & \multicolumn{2}{|c|}{ SF only $\quad(\alpha, n)$ only } & $\gamma$ only & Mix \\
\hline & & & & \\
\hline Singles & $230526 \pm 480$ & $536630 \pm 743$ & $90295 \pm 300$ & $856478 \pm 925$ \\
\hline Doubles & $67925 \pm 261$ & $25877 \pm 161$ & $4683 \pm 68$ & $98549 \pm 314$ \\
\hline Triples & $11715 \pm 108$ & $829 \pm 29$ & $24 \pm 5$ & $12328 \pm 111$ \\
\hline Quadruples & $1247 \pm 35$ & $21 \pm 5$ & & $1273 \pm 36$ \\
\hline
\end{tabular}

TABLE IV. CALCULATED COINCIDENCES FOR THE METALLIC MATRIX WITH A 5 CM THICK LEAD SCREEN, FOR A 25 MINUTES MEASUREMENT TIME AND $1 \mathrm{G}$ OF PU, WHEN PARALYZING THE TWO CLOSEST DETECTORS DURING 10 NS TO REJECT CROSS-TALK EVENTS.

\begin{tabular}{|c|c|c|c|c|}
\hline Source type $\rightarrow$ & SF only & $(\alpha, n)$ only & $\gamma$ only & Mix \\
\cline { 2 - 5 } Event type $\downarrow$ & \multicolumn{4}{|c|}{} \\
\hline Singles & $248585 \pm 499$ & $551464 \pm 743$ & $94067 \pm 307$ & $893234 \pm 945$ \\
Doubles & $55093 \pm 235$ & $11837 \pm 109$ & $935 \pm 31$ & $67703 \pm 260$ \\
Triples & $7322 \pm 86$ & $54 \pm 7$ & & $7281 \pm 85$ \\
Quadruples & $479 \pm 22$ & $2 \pm 1$ & & $479 \pm 21$ \\
\hline
\end{tabular}

TABLE V. CALCULATED COINCIDENCES FOR THE ORGANIC MATRIX WITH A 5 CM THICK LEAD SCREEN, FOR A 25 MINUTES MEASUREMENT TIME AND $1 \mathrm{G}$ OF PU, WITHOUT CROSS-TALK REJECTION.

\begin{tabular}{c|c|c|c|c|} 
Source type $\rightarrow$ & SF only & \multicolumn{4}{|c|}{$(\alpha$, n) only } & $\gamma$ only & Mix \\
\cline { 2 - 5 } Event type $\downarrow$ & $210006 \pm 458$ & $382725 \pm 619$ & $122416 \pm 350$ & $714561 \pm 845$ \\
Singles & $39285 \pm 198$ & $20850 \pm 144$ & $6192 \pm 79$ & $66629 \pm 258$ \\
Doubles & $4156 \pm 64$ & $724 \pm 27$ & $35 \pm 6$ & $4744 \pm 69$ \\
Triples & $296 \pm 17$ & $12 \pm 3$ & & $352 \pm 19$ \\
Quadruples & & & \\
\hline
\end{tabular}

TABLE VI. CALCULATED COINCIDENCES FOR THE ORGANIC MATRIX WITH A 5 CM THICK LEAD SCREEN, FOR A 25 MINUTES MEASUREMENT TIME AND $1 \mathrm{G}$ OF PU, WHEN PARALYZING THE TWO CLOSEST DETECTORS DURING 10 NS TO REJECT CROSS-TALK EVENTS.

\begin{tabular}{|c|c|c|c|c|}
\hline Source type $\rightarrow$ & SF only & $(\alpha, n)$ only & $\gamma$ only & Mix \\
\cline { 2 - 5 } Event type $\downarrow$ & \multicolumn{4}{|c|}{} \\
\hline Singles & $225680 \pm 475$ & $396257 \pm 629$ & $127454 \pm 357$ & $748882 \pm 865$ \\
Doubles & $26159 \pm 162$ & $8029 \pm 90$ & $1189 \pm 34$ & $35543 \pm 189$ \\
Triples & $1851 \pm 43$ & $25 \pm 5$ & & $1787 \pm 42$ \\
Quadruples & $76 \pm 9$ & & & $95 \pm 10$ \\
\hline
\end{tabular}


Analyzing the nature of triples associated to independent histories allows us to better understand the impact of crosstalk rejection for each matrix. As presented in Fig. 3, a major part of real triples of ${ }^{240} \mathrm{Pu}$ in the metallic matrix is constituted by three pulses of correlated fission neutrons (the so-called "nnn" coincidences), which do not lose much energy while traveling through the matrix, and thus are still detectable by plastic scintillators. By comparison, Fig. 4 shows a higher proportion of $\gamma \mathrm{nn}$ and $\gamma \gamma \mathrm{n}$ triples for ${ }^{240} \mathrm{Pu}$ in the organic matrix, since it partially moderates the fission neutrons. Indeed, the low energy electronics threshold used in the simulations is $100 \mathrm{keVee}$, thus rejecting neutrons with energy lower than $670 \mathrm{keV}$. One can note that $\gamma \mathrm{nn}$ and $\gamma \gamma \mathrm{n}$ coincidences involve fission prompt gamma rays with a high energy (several $\mathrm{MeV}$ ) crossing both the organic matrix and the $5 \mathrm{~cm}$ lead shield.

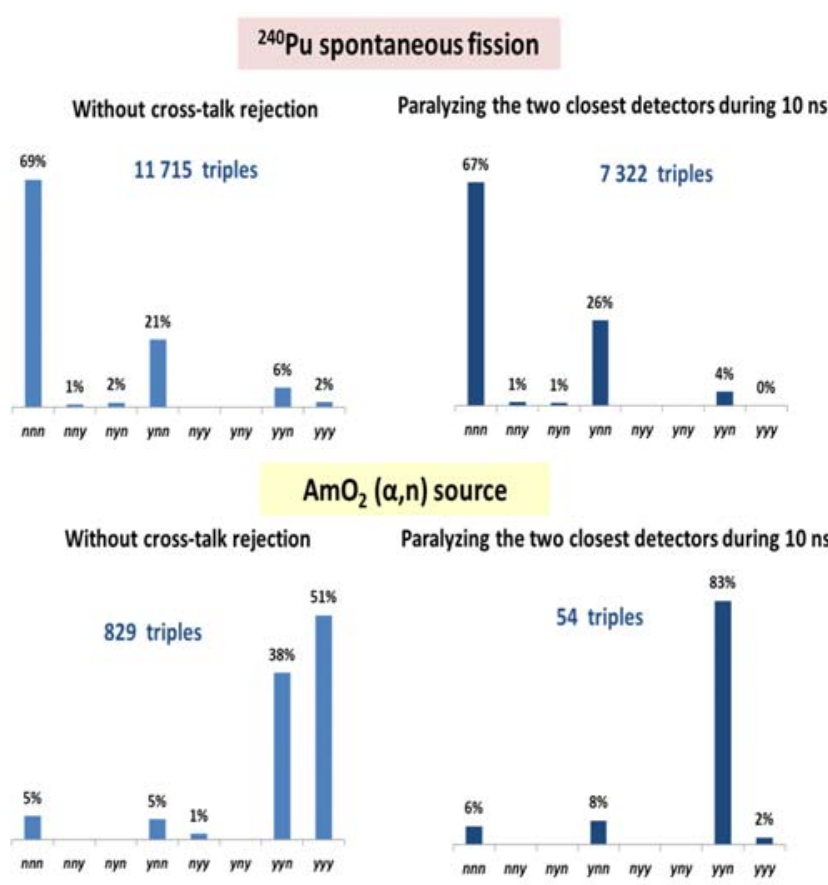

Fig. 3. Nature of real triples of ${ }^{240} \mathrm{Pu}$ and $(\alpha, \mathrm{n})$ reactions of $\mathrm{AmO}_{2}$ in the metallic matrix, without cross-talk rejection (on the left) and by rejecting the pulses of the closest neighboring detectors during $10 \mathrm{~ns}$ (on the right).

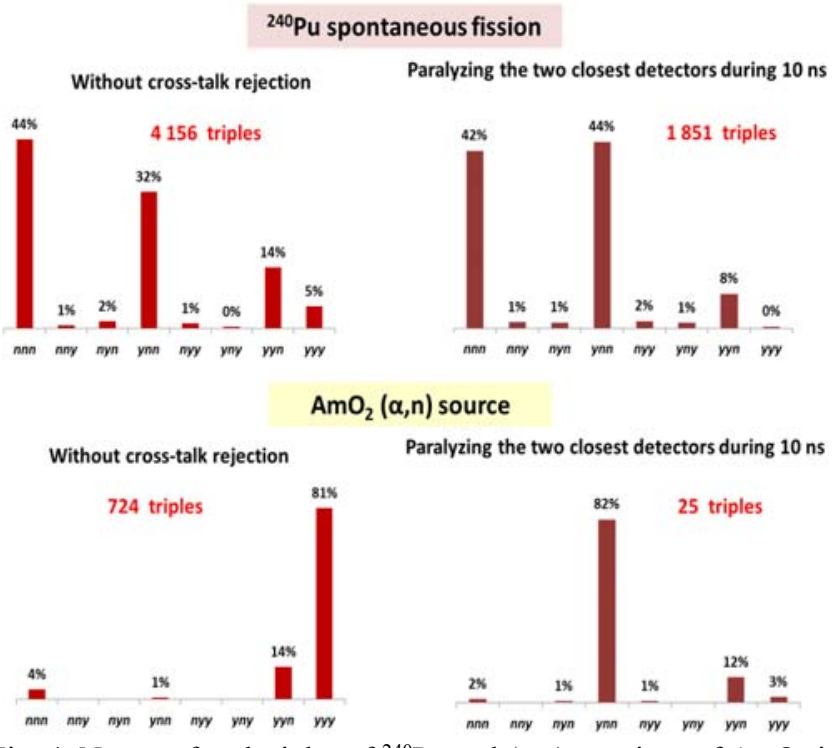

Fig. 4. Nature of real triples of ${ }^{240} \mathrm{Pu}$ and $(\alpha, n)$ reactions of $\mathrm{AmO}_{2}$ in the organic matrix, without cross-talk rejection (on the left) and by rejecting the pulses of the closest neighboring detectors during $10 \mathrm{~ns}$ (on the right).

Concerning parasitic triples induced by $(\alpha, n)$ reactions, gamma pulses are logically more numerous $(\gamma \gamma \mathrm{n}$ and $\gamma \gamma \gamma$ coincidences) in both matrices, because no correlated neutrons are emitted by the source contrary to spontaneous fission. Although some coincidences can be produced by inelastic scattering $\left(n, n^{\prime} \gamma\right)$, or by $(n, 2 n)$ reactions in lead, most of triples are due to gamma cross talk involving a capture gamma ray of hydrogen, or an energetic gamma ray emitted by the excited daughter nucleus of the $(\alpha, n)$ reaction, the $\mathrm{AmO}_{2}$ source of MCNPX PoliMi emitting prompt gamma rays in correlation with the $(\alpha, n)$ neutron, as explained in Section II, such as the $1634 \mathrm{keV}$ and $2438 \mathrm{keV}$ lines of ${ }^{20} \mathrm{Ne}$ and ${ }^{21} \mathrm{Ne}$, respectively. Concerning radiative capture, fast neutrons thermalized by elastic scattering on hydrogen nuclei of plastic scintillators, can finally be absorbed by $(\mathrm{n}, \gamma)$ reactions. Doubles or triples can then be produced in close detectors by the capture gamma ray of hydrogen $(2.225 \mathrm{MeV})$, due to Compton scattering or to correlated $511 \mathrm{keV}$ annihilation photons, following pair creation. Note that these pure gamma coincidences occurs after the moderation of the original $(\alpha, n)$ or fission fast neutron, which can last up to a few milliseconds. After such a long time, they are no more correlated with the original fast neutron interaction that opened the $100 \mathrm{~ns}$ coincidence window, and therefore they appear as independent gamma coincidences, the pulses of which being detected within a very short time (less than $1 \mathrm{~ns}$ ). The cross-talk rejection process consisting in freezing neighboring detectors during $10 \mathrm{~ns}$ efficiently discards such coincidences. Note that in Fig. 3 and Fig. 4, the triples due to cross-talk of radiative capture gamma rays are not taken in account. Indeed, only the particles detected less than $100 \mathrm{~ns}$ after the first pulse are taken into account for these plots. In the rest of the paper, however, coincidences due to capture gamma rays have been recorded, i.e. in any table or figure other than Fig. 3 and Fig. 4.

It can also be deduced from Tables II and III that, for the metallic matrix, cross-talk rejection reduces the counting 
statistics on ${ }^{240} \mathrm{Pu}$ triples by $37 \%$, while the reduction is much larger, $93 \%$, for the $\mathrm{AmO}_{2}(\alpha, n)$ source. In the organic matrix, the respective reduction proportions are $56 \%$ and $97 \%$, as deduced from Table IV and V. For both matrices, this efficient reduction of $\mathrm{AmO}_{2}$ triples is mainly due to the large suppression of $\gamma \gamma \gamma$ triples, as can be seen in Fig. 3 and Fig. 4. Before cross talk mitigation, the largest part of triples recorded with the $(\alpha, n)$ source was indeed due to gamma cross talk.

\section{INFLUENCE OF PLUTONIUM MASS}

In order to study the linearity of the PNCC response and the possible impact of accidental coincidences or neutron multiplication effects, the study has been extended to $\mathrm{Pu}$ masses ranging from $0.1 \mathrm{~g}$ to $100 \mathrm{~g}$. We study only the effect of $\mathrm{Pu}$ mass, using the MCNP PoliMi $\mathrm{AmO}_{2}$ alpha-source, thus considering plutonium in the $\mathrm{PuO}_{2}$ form, neglecting the $(\alpha, n)$ contribution due to ${ }^{240} \mathrm{Pu}$. The evolution of the number of coincidences as a function of $\mathrm{Pu}$ mass is reported in Fig. 5, showing a non-linearity of triples for large masses above $50 \mathrm{~g}$, relatively to the linear trend deduced from triples related to the plutonium masses between $0 \mathrm{~g}$ and $5 \mathrm{~g}$, which can be explained by induced fissions. For the maximum $\mathrm{Pu}$ mass of $100 \mathrm{~g}$, the excess of coincidences is about $9 \%$ for the metallic matrix, and $32 \%$ for the organic matrix in which more induced fissions occur. After cross-talk rejection, it reaches $12 \%$ and $44 \%$, respectively.

Concerning counting statistics uncertainties, all calculations are made here with a constant product of $\mathrm{Pu}$ mass and acquisition time, i.e. with a similar counting statistics. For instance, a long acquisition time of $15,000 \mathrm{~s}$ is simulated for the $0.1 \mathrm{~g} \mathrm{Pu}$ mass, while a short acquisition time of $15 \mathrm{~s}$ is used to limit processing time for the largest $\mathrm{Pu}$ mass of $100 \mathrm{~g}$. Note that the curves for quadruples are not reported because of large counting statistics uncertainties, especially after crosstalk rejection.

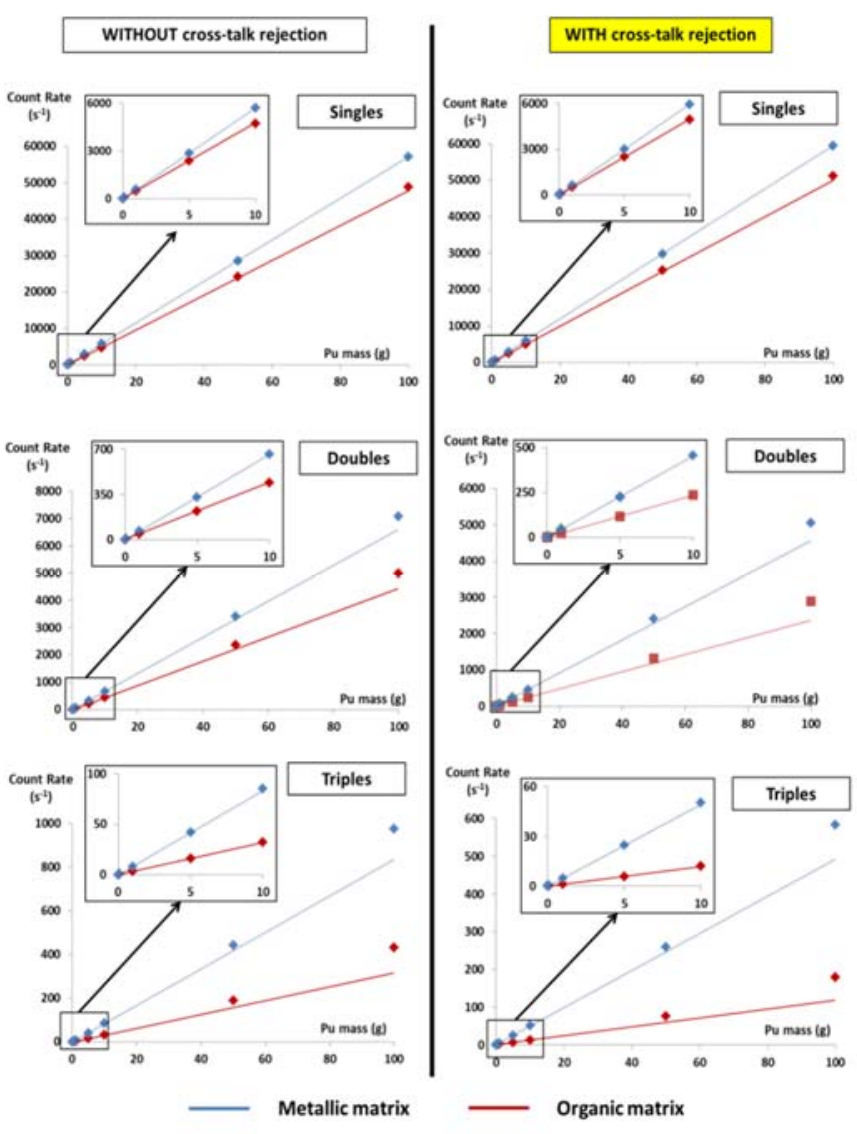

Fig. 5. Coincidence count rates of the mixed source $\left(\mathrm{Pu}, \mathrm{AmO}_{2}\right.$, gamma rays of $\mathrm{Pu}$ and $\mathrm{Am}$ ) as a function of Pu mass, without (left panels) and with (right panels) cross-talk rejection.

As discussed in previous section with $1 \mathrm{~g}$ of $\mathrm{Pu}$, the selectivity of the method to $\mathrm{Pu}$, with respect to $\mathrm{AmO}_{2}$ or other possible sources of correlated $(\alpha, n)$ neutrons and gamma rays, can be assessed by the fraction of triples due to spontaneous fissions of ${ }^{240} \mathrm{Pu}$ among the total amount of triples. Table VII shows that this fraction can be improved by the cross-talk rejection algorithm consisting in freezing the close neighbors of a primarily triggered detector during $10 \mathrm{~ns}$, but beyond $10 \mathrm{~g}$ of $\mathrm{Pu}$ it is no more possible to reach about $100 \%$ of useful triples, i.e. to reject almost entirely parasitic coincidences. This is due to fissions induced by $(\alpha, n)$ neutrons, which create coincidences that are logically not discarded by the cross-talk rejection algorithm, because they are similar to useful spontaneous fission coincidences.

TABLE VII. RATIO OF TRIPLES FROM THE ${ }^{240} \mathrm{PU}$ SOURCE TO THE TRIPLES FROM THE MIXED SOURCE $\left({ }^{240} \mathrm{PU}+\mathrm{AMO}_{2}+\gamma\right.$ RAYS OF PU AND AM), FOR DIFFERENT MASSES OF PU. WITH (IN BOLD SCRIPT) AND WITHOUT CROSS-TALK REJECTION (NORMAL SCRIPT)

\begin{tabular}{|c|rrrr|}
\hline Pu mass & $1 \mathrm{~g}$ & $10 \mathrm{~g}$ & $50 \mathrm{~g}$ & $100 \mathrm{~g}$ \\
\cline { 2 - 5 } Matrix & & & & \\
\hline
\end{tabular}




\begin{tabular}{|c|c|c|c|c|}
\hline \multirow{2}{*}{ Metallic } & $95.0 \% \pm 1.7 \%$ & $92.1 \% \pm 1.7 \%$ & $91.4 \% \pm 1.6 \%$ & $86.2 \% \pm 1.5 \%$ \\
& $\mathbf{1 0 0 . 0} \% \pm \mathbf{2 . 4} \%$ & $\mathbf{9 7 . 4} \% \pm \mathbf{2 . 3} \%$ & $\mathbf{9 7 . 4 \%} \pm \mathbf{2 . 2} \%$ & $\mathbf{8 9 . 5} \% \pm \mathbf{2 . 0} \%$ \\
\cline { 1 - 1 } Organic & $87.6 \% \pm 2.6 \%$ & $83.8 \% \pm 2.3 \%$ & $77 \% \pm 2.3 \%$ & $73.6 \% \pm 2.0 \%$ \\
& $\mathbf{1 0 0 . 0} \% \pm \mathbf{4 . 9} \%$ & $\mathbf{9 8 . 6} \% \pm \mathbf{4 . 6} \%$ & $\mathbf{8 4 . 9} \% \pm \mathbf{3 . 7} \%$ & $\mathbf{8 4 . 5 \%} \pm \mathbf{3 . 4} \%$ \\
\hline
\end{tabular}

\section{INFLUENCE OF PLUTONIUM LOCALIZATION}

The effect of $\mathrm{Pu}$ localization has also been studied for 3 point sources as shown in red on Fig. 6 or Fig. 7, in which the ring reflects the trajectory of an external point source inside a drum in rotation during acquisition. Compared to the reference homogeneous repartition, the number of triples and the proportion of useful $\mathrm{Pu}$ coincidences are not very sensitive to $\mathrm{Pu}$ position, whether for the $0.5 \mathrm{~g} . \mathrm{cm}^{-3}$ metallic matrix or for

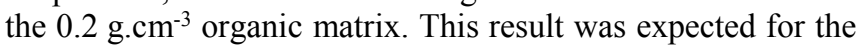
metallic matrix, which does not significantly slow-down spontaneous fission neutrons, but it is better than expected for the organic matrix, which is sufficiently light such that it does not moderate neutrons in a great extent. On the other hand, as already observed for the homogeneous distribution, cross-talk rejection allows removing almost entirely triples due to $(\alpha, n)$ reactions in both matrixes.

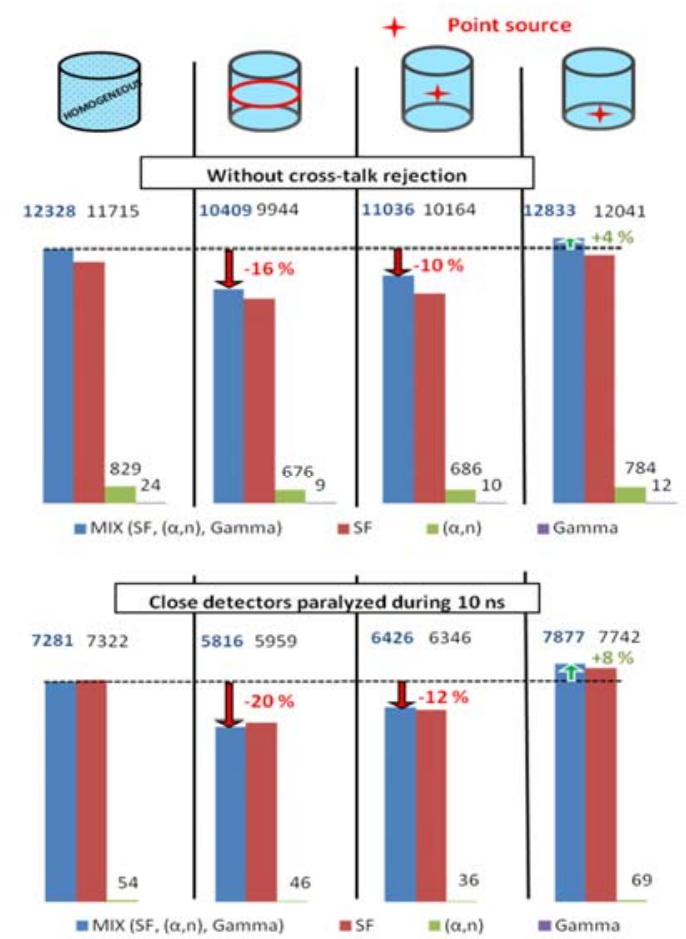

Fig. 6. Number of triples for the different sources (individually and mixed) as a function of $\mathrm{Pu}$ localization in a drum containing the metallic matrix and $1 \mathrm{~g}$ of $\mathrm{Pu}$, for a 25 min measurement, without (above) or with cross talk rejection (below).

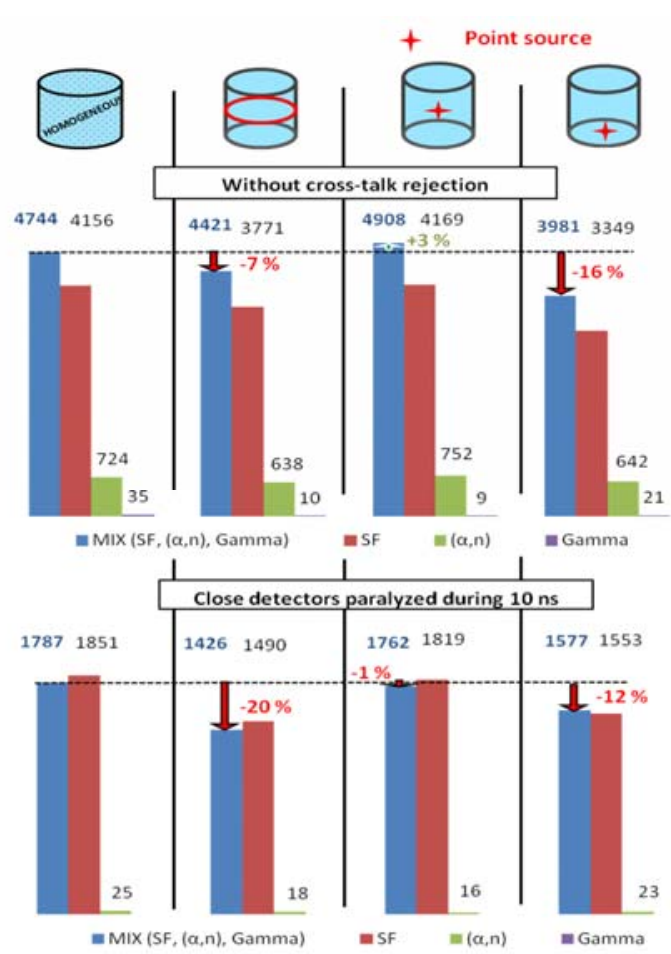

Fig. 7. Number of triples for the different sources (individually and mixed) as a function of $\mathrm{Pu}$ localization in a drum containing the organic matrix and $1 \mathrm{~g}$ of $\mathrm{Pu}$, for a $25 \mathrm{~min}$ measurement, without (above) or with cross talk rejection (below).

\section{CONCLUSION}

Simulations show that plastic scintillators can be used to measure plutonium by passive neutron coincidence counting in radioactive waste drums filled with metallic or organic technological wastes. A $5 \mathrm{~cm}$ lead screen is used in front of the detectors to cut most of the gamma rays emitted by the waste. Cross-talk rejection algorithms consisting in paralyzing the two closest neighbors of a primary triggered detector, during $10 \mathrm{~ns}$, increases to nearly $100 \%$ the fraction of $\mathrm{Pu}$ triples among the total number of triples. Indeed, the triples due to $(\alpha, n)$ reactions are composed of a majority of gamma pulses (mainly $\gamma \gamma \mathrm{n}$ or $\gamma \gamma \gamma$ coincidences), following gamma cross talk. Cross-talk rejection allows suppressing almost entirely this parasitic contribution with a $5 \mathrm{~cm}$ lead screen, as already observed with a $10 \mathrm{~cm}$ lead screen [7]. In addition, although cross-talk rejection significantly reduces the number of ${ }^{240} \mathrm{Pu}$ spontaneous fission triple coincidences, from nearly $40 \%$ in the metallic matrix to $60 \%$ in the organic one, this useful signal remains larger than 7000 counts for $1 \mathrm{~g}$ of $\mathrm{Pu}$ homogeneously distributed in the metallic matrix, after a 25 min measurement, and drop to 2000 counts in the organic matrix due to the presence of hydrogen. This latter matrix further reduces counting statistics because hydrogen nuclei moderate part of fission fast neutrons, making them undetectable by plastic scintillators. Nevertheless, these number of coincidences obtained with a $5 \mathrm{~cm}$ lead screen are significantly higher than with the previous $10 \mathrm{~cm}$ lead screen, by a factor 3.5 and 5 for the metallic and organic matrixes, respectively, indicating the influences of gamma rays in triple 
coincidences and of thickness of lead screen on the neutron detection efficiency.

The linearity of the system has also been studied up to $100 \mathrm{~g}$ of $\mathrm{Pu}$. For a few tens of $\mathrm{Pu}$ grams, the multiplication effect due to induced fissions generates additional coincidences, making the relation between the signal and the $\mathrm{Pu}$ mass no more linear above $50 \mathrm{~g}$. The resulting overestimation of the $\mathrm{Pu}$ mass, after cross talk rejection, would be about $12 \%$ for the maximum $\mathrm{Pu}$ mass of $100 \mathrm{~g}$ homogeneously distributed in the metallic matrix, and $44 \%$ in the organic matrix. We are currently studying the way to estimate the fraction of induced fission coincidences, for instance by analyzing the pulse train on the $\mu$ s scale, instead of only coincidences in the $100 \mathrm{~ns}$ time window.

Finally, we have also studied the effect of $\mathrm{Pu}$ localization in the waste matrix. A maximum $20 \%$ difference is observed on the number of triples between the homogeneous distribution and a point source placed in the periphery of the drum, for both the metallic and organic matrixes.

An experimental validation of the method has been undertaken with $10 \mathrm{~cm} \times 10 \mathrm{~cm} \times 100 \mathrm{~cm}$ plastic scintillators and a $118 \mathrm{~L}$ mock-up drum filled with ${ }^{252} \mathrm{Cf}$ and $\mathrm{AmBe}$ sources, or with $\mathrm{Pu}$ samples, and different metallic (small iron hollow tubes) and organic (small wood pieces) matrices.

\section{REFERENCES}

[1] D. Reilly, N. Ensslin, H. Smith Jr., Passive Nondestructive Assay of Nuclear Materials, NUREG/CR-5550, LA-UR-90-732, March 1991.

[2] R.T.Kouzes, The ${ }^{3}$ He Supply Problem (Technical Report,PNNL-18388), Pacific Northwest National Laboratory, Washington, 2009.

[3] M .J Joyce, K. A. Gamage, M. D. Aspinall, F. D. Cave, A. Lavietes, Real-Time, Fast Neutron Coincidence Assay of Plutonium With a 4Channel Multiplexed Analyzer and Organic Scintillators, IEEE Transactions on Nuclear Science, Vol. 61, N³, pp 1340-1348, 2014.

[4] C. C. Lawrence, M. Febbraro, T. N. Massey, M. Flaska, F. D. Becchetti, S. A. Pozzi, "Neutron response characterization for an EJ299-33 plastic scintillation detector," Nuclear Instruments and Methods in Physics Research Section A: Accelerators, Spectrometers, Detectors and Associated Equipment, vol. 759, pp. 16-22, Sep. 2014.

[5] J. M. Mueller and J. Mattingly, "Using anisotropies in prompt fission neutron coincidences to assess the neutron multiplication of highly multiplying subcritical plutonium assemblies," Nuclear Instruments and Methods in Physics Research Section A: Accelerators, Spectrometers, Detectors and Associated Equipment, vol. 825, pp. 87-92, Jul. 2016.

[6] C. Deyglun, C. Carasco B. Pérot, C. Eleon, G. Sannié, K. Boudergui, G. Corre, V. Kondrasovs, S. Normand, P. Pras, Passive and Active Correlation Techniques for the Detection of Nuclear Materials, IEEE Transactions on Nuclear Science, Vol. 61, N4, pp 2228-2233, 2014.

[7] B. Simony, C. Deyglun, B. Pérot, C. Carasco, N. Saurel, S. Colas, J. Collot, Cross-talk characterization in passive neutron coincidence counting of radioactive waste drums with plastic scintillators, IEEE Transactions on Nuclear Science, Vol. 63, ํ3, pp 1513-1519, 2016.

[8] E. Padovani, S. A. Pozzi, S.D. Clarke, E.C. Miller, MCNPX-PoliMi User's Manual, April 2012.

[9] S. A. Pozzi, E. Padovani, M. Marseguerra, MCNP-PoliMi : a MonteCarlo code for correlation measurements, Nuclear Instruments and Methods in Physics Research A 513 (2003) 550-558

[10] R. Brun, F. Rademakers, ROOT - An object oriented data analysis framework, NIM A 389 (1997) 81-86, http://root.cern.ch 